\title{
Composite Material for Supercapacitors Formed by Polymerization of Aniline in the Presence of Graphene Oxide Nanosheets
}

\author{
Y.M. Shulga ${ }^{1}{ }^{*}$, S.A. Baskakov ${ }^{1}$, V.V. Abalyaeva ${ }^{1}$, O.N. Efimov ${ }^{1}$, N.Y. Shulga ${ }^{2}$, \\ A. Michtchenko ${ }^{3}$, L. Lartundo-Rojas ${ }^{3}$, L.A. Moreno-Rojas 3 , J.G. Cabañas-Moreno ${ }^{3}$ \\ 1 Institute of Problems of Chemical Physics, Russian Academy of Sciences, \\ 142432 Chernogolovka, Moscow Region, Russia \\ 2 Moscow Steel and Alloys Institute, 117936 Moscow, Leninsky pr., 4 \\ 3 Centro de Nanociencias y Micro y Nanotecnologías Instituto Politécnico Nacional, \\ Luis Enrique Erro s / $n$ Zacatenco, 07738, Mexico City, Mexico
}

(Received 27 April 2012; published online 27 June 2012)

\begin{abstract}
The composite material was obtained by the polymerization of aniline in the presence of graphene oxide nanosheets (GONS). The resulting composite PANi (72\%) - GONS (28\%) was investigated by methods such as XPS, TGA, Raman and IR spectroscopy, and so on. It was established that a partial reduction of graphene oxide takes part in course of the polymerization. Specific capacitance of the PANi-GONS electrode in $1 \mathrm{M} \mathrm{H}_{2} \mathrm{SO}_{4}$, corresponding to its discharge from 0.700 to $0.052 \mathrm{~V}$, was found to be $547 \mathrm{~F} / \mathrm{g}$. But, if in the calculation of the capacitance include a shallow part of the discharge curve (below $0.15 \mathrm{~V}$ ), one can obtain the value of specific capacity greater than $1200 \mathrm{~F} / \mathrm{g}$.
\end{abstract}

Keywords: Graphene oxide nanosheets, Polyaniline, Supercapacitors, XPS, TGA, Raman and IR spectroscopy.

PACS number: 81.05.ue

\section{INTRODUCTION}

Number of open publications devoted to the study of different materials for electrodes of supercapacitors has been growing rapidly due to the discovery of new carbon nanostructures which have both high specific surface area and high conductivity and can possibly improve the performance of the devices.

In this paper, we present the results of the study of composition and structure of the composite obtained by polymerization of aniline in the presence of graphene oxide nanosheets (GONS). The composite shows specific capacity (SC) of $547 \mathrm{~F} / \mathrm{g}$. The main task of this study was to describe the mutual effect of components in the composite. It was also interesting to understand the origin of such a high spread of SC values for the PANIGONS composite.

\section{EXPERIMENTAL}

\subsection{Materials}

Graphite oxide was obtained as described in [1]. To prepare aqueous suspension of graphene oxide nanosheets, $300 \mathrm{mg}$ of graphite oxide was mixed with $400 \mathrm{ml}$ of distilled water and the mixture was treated in an ultrasonic bath for 1 hour. The resulting suspension was centrifuged for $15 \mathrm{~min}$ at $3000 \mathrm{~g}$ to remove large particles.

Synthesis of PANi-GONS composite. $1.07 \mathrm{~g}$ of aniline sulfate was dissolved in $50 \mathrm{ml}$ of distilled water. Suspension of GONS was added to the solution upon stirring. Then concentrated sulfuric acid was added in the reaction vessel to $\mathrm{pH}=2$. The mixture was cooled down to $-2 \pm 2{ }^{\circ} \mathrm{C}$ in an ice bath. Then $50 \mathrm{ml}$ of aqueous solution of ammonium persulfate was slowly added dropwise to the reaction in mixture at an equimolar ratio with aniline. The reaction proceeded for 4 hours in the above temperature range. The resulting precipitate was centrifuged and repeatedly washed with distilled water to form dark green pasty mass. The samples for electrochemical studies were prepared by deposition of this dark green substance on a glassy carbon plate of $0.5 \times 0.5 \times 4 \mathrm{~cm}$ followed by drying in air at $60-70^{\circ} \mathrm{C}$.

\subsection{Instruments}

X-ray photoelectron spectra (XPS) were measured with a Thermo Scientific K-Alpha spectrometer using monochromatic $\mathrm{Al} \mathrm{K}_{\alpha}$ radiation. Working pressure in the chamber of the spectrometer did not exceed $4 \times 10^{-10}$ Torr. The pass energy $(20 \mathrm{eV})$ was constant in all experiments. Raman spectra were excited using a laser with $\lambda=633 \mathrm{~nm}$. The spectra were recorded using LabRAM HR systems. Thermogravimetric analysis was performed on a Netzsch STA-409 instrument at $20-800^{\circ} \mathrm{C}$ at $10 \mathrm{deg} / \mathrm{min}$ heating rate. Cyclic voltammograms (CVA) and chargedischarge characteristics were measured in an electrochemical glass cell in $1 \mathrm{M} \mathrm{H}_{2} \mathrm{SO}_{4}$ in air at room temperature $\left(21-22^{\circ} \mathrm{C}\right)$. Working and auxiliary electrodes were separated by a porous glass membrane. An auxiliary electrode was glassy carbon plate $1 \times 4 \mathrm{~cm}$ in size and a reference electrode was $\mathrm{Ag} / \mathrm{AgCl}$ electrode. The measurements were performed with a PS -7 potentiostat.

\section{RESULTS AND DISCUSSION}

\subsection{IR spectra}

The presence of quinoid and benzoid rings in the composite is justified by the absorption bands (AB) at

*shulga@icp.ac.ru 
1568 and $1488 \mathrm{~cm}^{-1}$, respectively. As it derives from a comparison of intensity of these bands in the spectra of pure PANI and the composite that a relative share of quinoid structures increases at transition from pure PANI to the composite.

\subsection{XPS}

The analysis of shape of the N1s spectrum obtained by us showed no nitrogen of the imine type in the polymer. The main state is -NH- (more than 60\%). Positively charged nitrogen is also present in the sample. The fraction of such atoms (or the degree of doping of the polymer) is less than 0.4 .

The spectra of $\mathrm{C} 1 \mathrm{~s}$ of pure polyaniline and the composite shown in Fig. 1 are compared. The inset shows the difference spectrum obtained by subtracting the spectrum of PANI from that of the composite as described in [45]. The difference spectrum corresponds to the $\mathrm{C} 1 \mathrm{~s}$ spectrum of graphene oxide nanosheets, which are present in the composite.

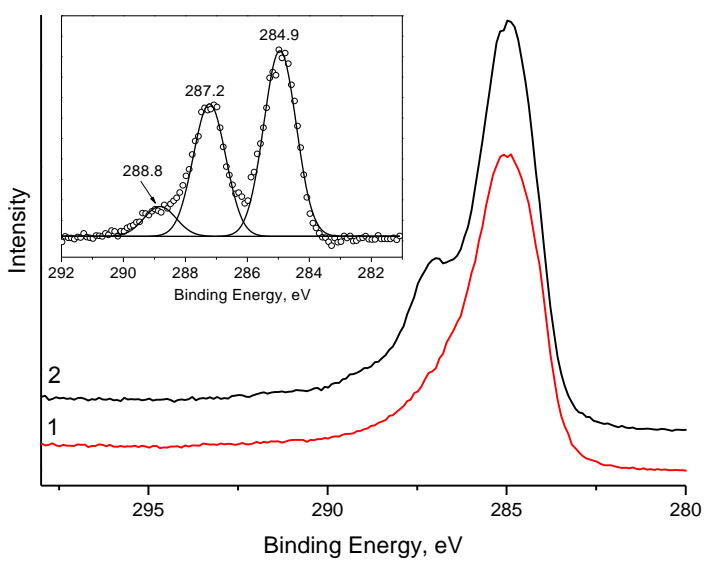

Fig. 1 - C1s spectra of polyaniline (1) and PANI- GONS composite (2). Intensities of the spectra are calibrated on intensity of the N1s peak. The inset shows the spectrum obtained by subtracting spectrum 1 from spectrum 2

The difference spectrum is well approximated by 3 Gaussians. The peak with $E_{b}=284.9 \mathrm{eV}$ is due to the carbon atoms closely surrounded by other carbon atoms only. The second peak $(287.2 \mathrm{eV})$ is attributed by most authors to carbon atoms singly bonded with an oxygen atom, i. e. epoxy $(>\mathrm{COC}<$ ) and/or hydroxyl (> C-OH) groups. The third peak can be attributed to carboxyl $(\mathrm{COOH})$ groups, i.e. carbon atoms bonded by 2 bonds with oxygen atoms. Comparing the intensities of individual peaks, one can conclude on that $37 \%$ of the carbon atoms of graphene oxide nanosheets are bonded by one bond with oxygen atom, and $15 \%$ are bonded by two bonds with carbon atoms in the composite.

The C1s spectrum of initial GO nanosheets differs from that of the composite (Fig. 2). Decomposition

\section{REFERENCE}

1. V.E. Muradian, M.G. Ezernitskaya, V.I. Smirnova, N.M. Kabaeva, M.E. Volpin, J. General Chem. 61, 2626 (1991). to individual components showed that $46 \%$ of the carbon atoms in the initial GONS are bonded with one oxygen atom, and 9\% - with two carbon atoms. Thus, GONS partially reduce in course of polymerization.

\section{$3.3 \quad$ CVA}

The analysis of the CVA curves of the composite film showed that specific capacity of the freshly prepared film is not very high. It varies from 20 to $100 \mathrm{~F} / \mathrm{g}$. To increase specific capacity can be enhanced using a special procedure of aging the electrode covered by film at low negative potentials. Fig. 8 shows the discharge curve for such an electrode. Electrode capacity was calculated according to the formula: $C=I t / P \Delta E$, where $I-$ discharge current, $t$-time of discharge, $P$-sample weight, $\Delta E$-working potential range. $\mathrm{SC}$ corresponding to the discharge to $0.052 \mathrm{~V}$ was found to be $547 \mathrm{~F} / \mathrm{g}$. It should be noted that if in the calculation of capacity includes only a flat part of the curve (below $0.15 \mathrm{~V}$ ), the value of specific capacity higher than $1500 \mathrm{~F} / \mathrm{g}$ is obtained. It is clear that capacitors with such small voltages are not interesting for practical use. However, the presence of the flat part in the discharge curve indicates that this material can be used as a supercapacitor electrode if better conditions for charge removal are provided. The CVA curve for the electrode under study also indicates its high specific capacity.

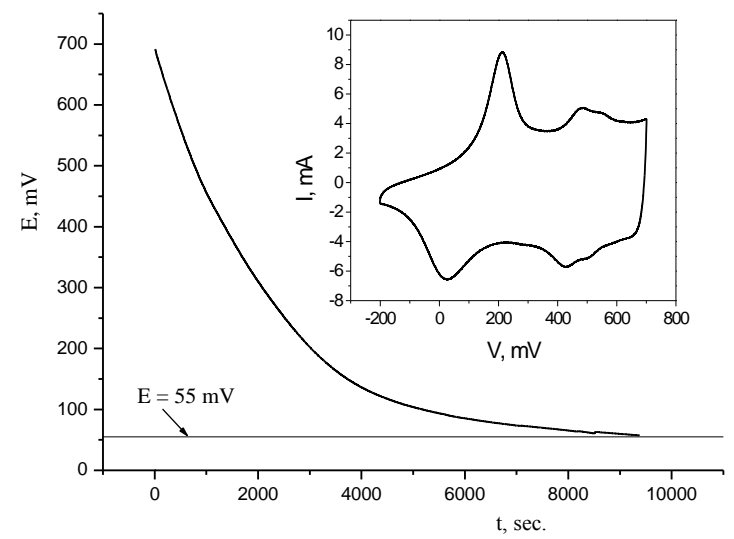

Fig. 2 - The discharge curve $(i=0.01 \mathrm{~mA})$ in $1 \mathrm{M} \mathrm{H} 2 \mathrm{SO} 4$ for the PANi-GONS electrode with mass $0.28 \mathrm{mg}$. The inset shows a typical CVA curve for this electrode

We note here that if the capacitance calculated from the formula $d E / d t=I / C$, the maximum specific capacitance reaches $1800 \pm 600 \mathrm{~F} / \mathrm{g}$.

\section{Acknowledgment}

The work is supported by Russian Foundation for Basic Research (Project 12-03-00261-a).

2. N.Y. Shulga, E.A. Skryleva, S.A. Baskakov, Y.M. Shulga, J. Experimental Nanosci. (2012) (in press). 